\title{
Exercise-induced AMPK activation does not interfere with muscle hypertrophy in response to resistance training in men
}

\author{
Tommy R. Lundberg, ${ }^{1}$ Rodrigo Fernandez-Gonzalo, ${ }^{2,3}$ and Per A. Tesch ${ }^{2}$ \\ ${ }^{1}$ Department of Health Sciences, Mid Sweden University, Östersund, Sweden; ${ }^{2}$ Department of Physiology and Pharmacology, \\ Karolinska Institutet, Stockholm, Sweden; and ${ }^{3}$ Department of Laboratory Medicine, Section for Clinical Physiology, \\ Karolinska Institutet, Karolinska University Hospital, Stockholm, Sweden
}

Submitted 26 September 2013; accepted in final form 8 January 2014

\begin{abstract}
Lundberg TR, Fernandez-Gonzalo R, Tesch PA. Exercise-induced AMPK activation does not interfere with muscle hypertrophy in response to resistance training in men. J Appl Physiol 116: 611-620, 2014. First published January 9, 2014; doi:10.1152/japplphysiol.01082.2013.—As aerobic exercise (AE) may interfere with adaptations to resistance exercise (RE), this study explored acute and chronic responses to consecutive $\mathrm{AE}(\sim 45$ min cycling $)$ and $\mathrm{RE}(4 \times 7$ maximal knee extensions) vs. RE only. Ten men performed acute unilateral $\mathrm{AE}+$ $\mathrm{RE}$ interspersed by $15 \mathrm{~min}$ recovery. The contralateral leg was subjected to RE. This exercise paradigm was then implemented in a 5 -wk training program. Protein phosphorylation, gene expression, and glycogen content were assessed in biopsies obtained from the vastus lateralis muscle of both legs immediately before and $3 \mathrm{~h}$ after acute RE. Quadriceps muscle size and in vivo torque were measured, and muscle samples were analyzed for citrate synthase activity and glycogen concentration, before and after training. Acute AE reduced glycogen content $(32 \% ; P<0.05)$ and increased $(P<0.05)$ phosphorylation of AMPK (1.5-fold) and rpS6 (1.3-fold). Phosphorylation of p70S6K and 4E-BP1 remained unchanged. Myostatin gene expression was downregulated after acute $\mathrm{AE}+\mathrm{RE}$ but not RE. Muscle size showed greater $(P<0.05)$ increase after $\mathrm{AE}+\mathrm{RE}(6 \%)$ than $\mathrm{RE}$ $(3 \%)$ training. Citrate synthase activity $(18 \%)$ and endurance performance $(22 \%)$ increased $(P<0.05)$ after AE + RE but not RE. While training increased $(P<0.05)$ in vivo muscle strength in both legs, normalized and concentric torque increased after RE only. Thus AE activates AMPK, reduces glycogen stores, and impairs the progression of concentric force, yet muscle hypertrophic responses to chronic RE training appear not to be compromised.
\end{abstract}

aerobic exercise; gene expression; human skeletal muscle signaling; muscle strength and power

IF PRECEDED BY AEROBIC EXERCISE (AE), typical outcomes of resistance exercise (RE) training, e.g., increased strength, power, and muscle size, may be compromised $(30,37)$. While the requisites and associated mechanisms of such interference have not been elucidated, residual fatigue and antagonistic molecular responses derived from previous exercise have been put forth as tentative causes to such an effect $(27,40)$. However, when allowing for $6 \mathrm{~h}$ recovery between bouts, we recently reported that $5 \mathrm{wk}$ concurrent $\mathrm{AE}+\mathrm{RE}$ training produced similar improvements in muscle strength and power, and even greater increases in muscle size, as RE alone (42). Thus time allowed for recovery between bouts may be vital in optimizing muscle adaptations to $\mathrm{AE}+\mathrm{RE}$ training.

It is well founded that acute AE reduces muscle glycogen content $(13,29)$ and may compromise neuromuscular function

Address for reprint requests and other correspondence: T. R. Lundberg, Dept. of Health Sciences, Mid Sweden Univ., 83125 Östersund, Sweden (e-mail: tommy.lundberg@miun.se).
$(5,36)$. Thus undertaking RE immediately after AE may impair muscle strength and power (39). Apart from interfering with in vivo function, decreased glycogen availability may also directly or indirectly impact aspects of muscle signaling following RE (17). Although this notion has been challenged (12), collectively, it appears that acute AE induces fatigue that could blunt the desired response to subsequent RE and hence attenuate muscle adaptations to chronic RE training.

At the cellular level, AE upregulates adenosine monophosphate-activated protein kinase (AMPK) to restore AMP/ATP balance and to trigger transcriptional activators, regulating mitochondrial biogenesis (14). RE increases protein synthesis through the mammalian target of rapamycin (mTOR) signaling pathway, and hence, cumulative RE favors contractile protein accretion (45). In support of this stereotypic mode-specific response, rat muscles subjected to either high- or low-frequency electrical stimulation purported to mimic RE and AE, respectively, showed increased $\mathrm{MTOR}$ phosphorylation after RE but not AE (3). Conversely, AE hyperphosphorylated AMPK and increased protein levels of the peroxisome proliferator-activated receptor- $\gamma$ coactivator-1 (PGC-1 $\alpha$ ) (3). While those findings imply that mode-specific signaling through AMPK and mTOR pathways dictates classical end-point adaptations to chronic $\mathrm{AE}$ and $\mathrm{RE}$ training, results of human studies are equivocal. For example, when strength- and endurance-trained athletes performed their habitual or nonhabitual exercises, robust anabolic muscle signaling was noted after unaccustomed exercise only (16). As reports also suggest AMPK increases in response to RE (20) and mTOR to be activated by AE (43), it appears important signaling routes dictating human skeletal muscle adaptations are shared for different exercise modes.

In the rat, contraction-induced mTOR signaling is inhibited by prior AMPK activation caused by endurance exercise (60). More specifically, translational signaling is compromised through attenuation of key downstream regulators such as eukaryotic initiation factor 4E-binding protein (4E-BP1) and p70S6 kinase (p70S6K) (60). This is in concert with the marked suppression of protein synthesis following AMPK activation (8). Collectively, this scenario offers reasonable mechanistic support for myofiber protein accretion to be blunted, as noted after concurrent $\mathrm{AE}$ and RE training (27).

In human subjects who performed $\mathrm{AE}$ prior to RE (15), the muscle anabolic response was somewhat attenuated compared with exercise performed in the reversed order. Indeed, performing RE followed by AE shows neither molecular interference (2) nor altered rate of protein synthesis (19) compared with $\mathrm{RE}$ alone. Our recent studies, examining responses to $\mathrm{AE}$ followed by RE, showed uncompromised translational signal- 
ing and muscle hypertrophy when recovery to restore muscle function between bouts was allowed $(41,42)$. Given that acute $\mathrm{AE}$ transiently impairs muscle function (5), reduces glycogen stores (36), and activates AMPK (11), it may be that back-toback $\mathrm{AE}+\mathrm{RE}$ interferes with translational signaling and hence attenuates muscle hypertrophy following chronic training.

Therefore, the aim of the current study was to investigate acute and chronic outcomes of consecutive $\mathrm{AE}+\mathrm{RE}$ and training compared with RE alone. It was hypothesized that 1 ) acute AE would reduce muscle glycogen stores and activate AMPK signaling, 2) AE would compromise RE-induced mTOR signaling, and 3) acute and chronic AE + RE would impair in vivo contractile function and blunt muscle hypertrophy compared with RE alone.

\section{METHODS}

General design. Ten men performed unilateral consecutive bouts of knee extensor AE ( $\sim 45 \mathrm{~min})$ and $\mathrm{RE}$ ( 4 sets of 7 reps) interspersed by 15 min recovery. The contralateral limb was subjected to RE only. Analysis of vastus lateralis muscle biopsies, obtained in both legs before (PRE) and $3 \mathrm{~h}$ after (POST) RE (Fig. 1), measured glycogen concentration, gene expression, and protein phosphorylation. These two exercise regimens were subsequently implemented into a 5-wk training program. Before and after training, muscle strength and power were assessed and quadriceps femoris (QF) muscle volume, cross-sectional area (CSA), and signal intensity (SI) determined by means of magnetic resonance imaging (MRI). Resting muscle biopsies assessed training-induced changes in citrate synthase (CS) activity and glycogen content.

Subjects. Ten healthy male volunteers $(26 \pm 5 \mathrm{yr}, 183 \pm 7 \mathrm{~cm}$, and $77 \pm 9 \mathrm{~kg}$ ) completed the study protocol. Subjects were moderately trained college students performing recreational exercise $\sim 3$ days/wk. While most subjects had practiced RE training at some time, none had performed structured weight training in the past year prior to the study. The study experiments and procedures were explained before subjects gave their written informed consent to participate. The study was approved by the Regional Ethical Review Board in Stockholm.

Exercise equipment. AE was performed using a modified onelegged cycle ergometer (model 828E, Monark Exercise AB, Varberg, Sweden) as previously described $(1,42)$. Open-chain concentric $(\mathrm{CON})$ knee extensions were performed at a target cadence of $60 \mathrm{rpm}$. Power and cadence were sampled at $2 \mathrm{~Hz}$ (SRM GmbH, Jülich, Germany). RE was performed using a seated knee extension ergometer (YoYo Technology, Stockholm, Sweden) equipped with a flywheel offering inertial resistance $\left(0.11 \mathrm{~kg} / \mathrm{m}^{2}\right)$ during coupled CON and eccentric (ECC) muscle actions (59). Peak torque and power were calculated from force (MuscleLab, Langesund, Norway) and speed (SmartCoach, Stockholm, Sweden) measurements sampled at $100 \mathrm{~Hz}$. The coefficient of variation $(\mathrm{CV} \%)$ for assessing flywheel peak power across two different sessions within $1 \mathrm{wk}$ was $5 \%$. Maximal isometric

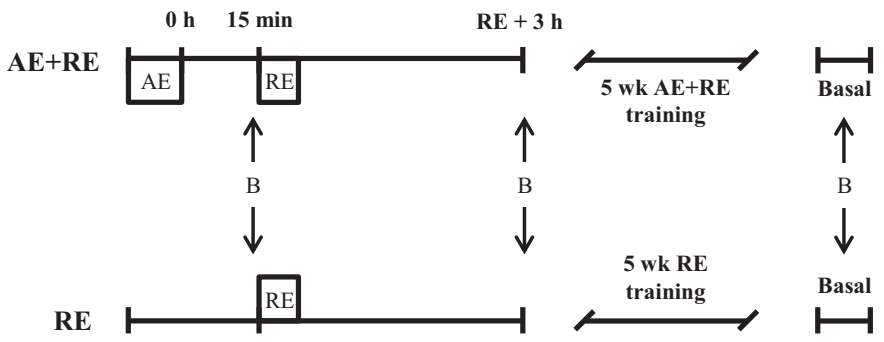

Fig. 1. Schematic overview of the study protocol. AE, aerobic exercise; RE, resistance exercise; B, muscle biopsy. and isokinetic torque were assessed using a Cybex II (Lumex, New York) dynamometer employing protocols described elsewhere (42). For all apparatuses used, individual settings were maintained throughout the study. Subjects completed three familiarization sessions in the course of 2 wk prior to the study. Standardized warm-ups preceded any exercise test or training session.

Acute exercise bouts. The acute exercise experiment was performed $1 \mathrm{wk}$ prior to commencing $5 \mathrm{wk}$ training. First, one randomly chosen limb completed 40 -min one-legged $\mathrm{AE}$ at $\sim 70 \%$ of maximal workload $\left(\mathrm{W}_{\max }\right)$ using a cadence of $60 \mathrm{rpm}$. To ensure strenuous efforts, ratings of perceived exertion (RPE; central and local) were obtained using the 6-20 Borg scale (9). At $40 \mathrm{~min}$, the workload was increased by $\sim 20 \mathrm{~W}$ and subjects were requested to continue until failure. Heart rate was recorded continuously (Polar Electro, Kempele, Finland). Fifteen minutes after completing AE, unilateral RE $(4 \times 7$ maximal repetitions; 2 min rest between sets) was executed for each leg in a random order on the knee extension ergometer. Subjects were verbally encouraged and requested to perform each repetition at maximal effort. Peak power was measured in each repetition.

Training protocols. The $\mathrm{AE}+\mathrm{RE}$ training regimen was dedicated to the leg performing $\mathrm{AE}+\mathrm{RE}$ during the acute experiments. The other limb served as control and performed RE only. Subjects completed $15 \mathrm{AE}$ sessions (3 nonconsecutive days/wk) and 12 unilateral RE sessions for both limbs (2 days/wk during weeks 1, 3, and 5 and 3 days/wk during weeks 2 and 4 ). Thus any RE session (i.e., $4 \times 7$ knee extensions for each leg) was preceded by AE for the intervention leg, and allowed for 15 min recovery between bouts. All AE and RE training sessions were supervised.

Muscle biopsies and diet control. Muscle biopsies were obtained from the mid portion of vastus lateralis under local anesthesia immediately before (PRE) and $3 \mathrm{~h}$ after (POST) the acute RE bout of either leg (Fig. 1). The $3 \mathrm{~h}$ time point was chosen to accommodate for changes in both protein phosphorylation and gene expression. In addition, resting muscle samples from either leg were collected $72 \mathrm{~h}$ after the last training session. Five millimeter Bergström-needles (7) with suction applied were used to obtain $\sim 150 \mathrm{mg}$ tissue samples that were cleansed of excess blood, fat, and connective tissue before being frozen in liquid nitrogen-precooled isopentane and stored at $-80^{\circ} \mathrm{C}$. Subsequent biopsies were obtained from separate incisions, moving in direction distal to proximal. A standardized dinner (pasta, tomato sauce, and juice) consisting of $2.21 \mathrm{~g}$ carbohydrates $/ \mathrm{kg}$ body wt (bw), $0.22 \mathrm{~g}$ protein $/ \mathrm{kg} \mathrm{bw}$, and $0.04 \mathrm{~g}$ fat $/ \mathrm{kg}$ bw was provided the night before experiments. On days of scheduled biopsies, a liquid formula (1.01 g carbohydrates $/ \mathrm{kg}$ bw, $0.31 \mathrm{~g}$ protein $/ \mathrm{kg}$ bw, and $0.24 \mathrm{~g} \mathrm{fat} / \mathrm{kg}$ bw) was provided as breakfast $2 \mathrm{~h}$ prior to the first biopsy. The liquid formulas contained $6.3 \mathrm{~g}$ protein (0.55 g leucine), $20.2 \mathrm{~g}$ carbohydrates, and $4.9 \mathrm{~g}$ fat per $100 \mathrm{ml}$ (Ensure Plus, Abbott Laboratories, Maidenhead, UK).

Pre- and posttesting. MRI scans (see below) were scheduled prior to any test or biopsy. Three or four days after acute experiments, maximal isometric and isokinetic strength, knee extension torque and power, and one-legged endurance performance were assessed over 2 days. Muscle strength and power for the right leg and endurance for the left leg were measured on day 1. The order of tests was reversed on day 2. Measures of peak torque were obtained at constant velocities of $0.52,1.05,2.09,3.14,3.67$, and $4.19 \mathrm{rad} / \mathrm{s}$ using the Cybex II dynamometer. Subjects performed $2-3$ attempts $(30$ s rest) at each velocity and the best result represented peak torque. Maximal isometric torque was measured at knee angle $120^{\circ}$. Subjects were instructed to push with maximal effort for $\sim 5 \mathrm{~s}$. The best score (two trials) in a $1 \mathrm{~s}$ window defined peak isometric torque. Subsequently, peak torque and power were assessed on the flywheel knee extension ergometer. Subjects performed $2 \times 7$ repetitions ( 2 min rest between sets) under strong verbal encouragement. Peak values were averaged across sets and repetitions, and normalized torque $\left(\mathrm{N} \cdot \mathrm{m} / \mathrm{cm}^{2}\right)$ was calculated as the ratio between peak knee extension torque and average muscle CSA. Finally, the one-legged ergometer incremental test assessed 
$\mathrm{W}_{\max }$ and endurance performance. Resistance was increased by $2.5 \mathrm{~N}$ every 2 nd minute until failure to maintain cadence. $\mathrm{W}_{\max }$ was defined as the last successfully completed workload. Heart rate was recorded continuously throughout the test. RPE was obtained every 2 nd minute and at exhaustion. Postperformance tests were completed identical to the pretests at the same time of the day $( \pm 2 \mathrm{~h})$. Subjects were blind to any test result to ensure nonbiased efforts. The post-MRI scans were obtained $48-72 \mathrm{~h}$ after the last training session. Resting muscle biopsies were obtained within 2 days after the MRI scans. Throughout the study, subjects were instructed to maintain ordinary daily routines, yet to refrain from strenuous activities involving the lower limbs.

Magnetic resonance imaging. Subjects rested in the supine position for $1 \mathrm{~h}$ prior to any MRI scan (6). Cross-sectional T2 weighted images were obtained using a 1.5-Tesla Philips MR Systems Intera (Best, The Netherlands) unit as previously described (42). During each scan, 50 images with $10-\mathrm{mm}$ slice thickness were obtained. Anatomical landmarks and standardized procedures ensured that the same segment was scanned before and after training. CSA and SI (mean gray value; MGV) of each individual QF muscle [vastus lateralis (VL), vastus intermedius (VI), vastus medialis (VM), and rectus femoris (RF)] were analyzed from the image where gluteus maximus was no longer visible, to the last image in which RF appeared. Every third image was analyzed to quantify CSA and SI using ImageJ software (National Institutes of Health, Bethesda, MD). As an additional control, SI of the biceps femoris (BF) muscle was analyzed in the third image of each subject. Mean CSA was multiplied by slice thickness to obtain muscle volume. In our laboratory, the $\mathrm{CV}$ for interindividual assessment of muscle volume amounts to $1 \%$.

CS activity and glycogen content. Freeze-dried muscle samples ( $\sim 3 \mathrm{mg}$ ) were homogenized in phosphate buffer with $0.5 \%$ BSA. CS activity and glycogen content were subsequently determined in duplicates through fluorometric assays as described elsewhere (42).

RNA isolation, reverse transcription, and real-time PCR. Gene expression of established markers of muscle adaptations to $\mathrm{AE}$ and $\mathrm{RE}$ were analyzed. Twenty-milligram muscle samples were homogenized using TRIzol and total RNA was extracted. One microgram of total RNA from each sample was used for reverse transcription into cDNA for a final volume of $20 \mu \mathrm{l}$ (High Capacity Reverse Transcription Kit, Applied Biosystems, Foster City, CA). Real-time PCR (ABI-PRISMA 7700 Sequence Detector, Perkin-Elmer Applied Biosystems) procedures were employed to determine mRNA expression. Probes and primers (TaqMan) for atrogin-1 (Hs00369714_m1), Muscle RING-finger protein-1 (MuRF-1; Hs00822397_m1), myostatin (Hs00193363_m1), PGC-1 $\alpha$ (Hs01016724_m1), and vascular endothelial growth factor (VEGF; Hs99999070_m1) were purchased from Applied Biosystems. GAPDH (Hs99999905_m1) and 18S (Hs01375212 g1) were used as reference genes. The expression of reference genes did not differ across time points and the GAPDH/18S ratio did not change. Reaction and amplification mixes $(10 \mu \mathrm{l})$ consisted of the diluted $(1: 100)$ cDNA $(4.5 \mu \mathrm{l})$, TaqMan Fast Universal PCR Master Mix $(5.0 \mu \mathrm{l})$, and specific primers $(0.5 \mu \mathrm{l})$. Subsequent cycling protocols were $2 \mathrm{~min}$ at $50^{\circ} \mathrm{C}$ and $10 \mathrm{~min}$ at $90^{\circ} \mathrm{C}$ followed by 40 cycles at $95^{\circ} \mathrm{C}$ for $15 \mathrm{~s}$ and $60^{\circ} \mathrm{C}$ for $1 \mathrm{~min}$. Target gene expression was reported as a ratio to the average of the two reference genes using the $2^{-\Delta \mathrm{CT}}$ formula.

Protein extraction and western blotting. About $30 \mathrm{mg}$ muscle tissue was manually homogenized in RIPA buffer, and proteins were recovered as previously described (41). Protein concentrations were subsequently determined using the Bradford technique. Thirty micrograms of protein per sample were loaded on $10 \%$ SDS precast gels (Bio-Rad) and separated through electrophoresis together with a protein ladder. Gels were transferred to PVDF-membranes using the Trans-Blot Turbo Transfer System from Bio-Rad. Blocking was completed using fluorescent blocking buffer (Millipore, Billerica, MA) during $60 \mathrm{~min}$ at room temperature (RT). Membranes were incubated overnight at $4^{\circ} \mathrm{C}$ with primary antibodies $(1: 1,000)$ for phospho-P70S6K (Thr389), phospho-rpS6 (Ser235/236), phospho-4E-BP1 (Thr37/46), and phos-
pho-AMPK $\alpha$ (Thr172). All antibodies were from Cell Signaling Technology (Beverly, MA), except for the antibody against p70S6K which was from Santa Cruz Biotechnology (Santa Cruz, CA). After the overnight incubation, membranes were washed $(4 \times 5 \mathrm{~min})$ in PBST $(0.1 \%)$ and incubated with IRDye secondary antibody (LI-COR Biosciences, Cambridge, UK) for $60 \mathrm{~min}$ at RT. A final series of washes were then performed before scanning the membranes (Odyssey SA Infrared Imaging System, LI-COR Bioscience). The blots were subsequently quantified using ImageJ. To control for loading error, phosphorylated proteins were expressed relative to total $\alpha$-tubulin abundance (1:20,000; Sigma-Aldrich, St. Louis, MO).

Data analysis. Dependent variables were analyzed by two-way repeated-measures ANOVA (factors: time and leg). When CON and ECC muscle actions were analyzed separately, three-way ANOVAs were employed. CS activity was analyzed by one-way ANOVA over time. Data skewness was assessed through histograms and the Shapiro-Wilk test. Positively skewed variables (PGC-1 $\alpha$ and VEGF) were log-transformed. When two-way interaction was found significant, a priori planned simple effect comparisons within each level were performed. The false discovery rate (FDR) procedure was employed to adjust for these comparisons (18). Significance was accepted at the $5 \%$ level $(P<0.05)$. Data are presented as means \pm SD.

\section{RESULTS}

Acute exercise experiment. Average power during $40 \mathrm{~min}$ $\mathrm{AE}$ was $37 \pm 6 \mathrm{~W}$. Power increased to $60 \pm 10 \mathrm{~W}$ during the final increment to exhaustion, which lasted 2 min $\pm 12 \mathrm{~s}$. RPE rose in a linear fashion, to attain 13 (central) and 15 (local) during the final $10 \mathrm{~min}$ of exercise, and 14 and 18 at exercise completion. Average HR during AE was $118 \pm 18$ beats $/ \mathrm{min}$. $\mathrm{HR}$ amounted to $148 \pm 16$ beats/min during the final exercise stage, and peaked at $161 \pm 14$ beats/min. In the subsequent $\mathrm{RE}$, the leg that had completed AE showed 10\% lower (351 \pm $97 \mathrm{~W} ; P=0.020)$ peak power than the leg subjected to RE only $(387 \pm 88 \mathrm{~W})$.

Aerobic exercise training. Average power across the $15 \mathrm{AE}$ sessions was $46 \pm 13 \mathrm{~W}$. Average power amounted to $39 \pm 7$ $\mathrm{W}$ in the first and increased $(P=0.004)$ to $53 \pm 15 \mathrm{~W}$ during the last session. At the final incremental step to exhaustion (2 $\min \pm 25 \mathrm{~s}$ ), power averaged $66 \pm 18 \mathrm{~W}$. RPE (local) increased over the course of exercise to attain near maximal values $(19 \pm 1)$ at exercise completion. HR averaged $120 \pm 17$ beats/min during the 40-min sessions and increased to $146 \pm$ 21 beats/min during the final exercise stage. Average peak HR across training sessions was $160 \pm 18$ beats $/ \mathrm{min}$.

Resistance exercise training. Changes in peak power for $\mathrm{CON}$ and ECC muscle actions were similar over the 5-wk training; hence CON-ECC data were merged. There was no leg $\times$ time interaction for peak power. Thus the progression in power across RE training sessions (main effect of time, $P<0.0005$; Fig. 2) was comparable for the different legs. However, the leg subjected to $\mathrm{AE}+\mathrm{RE}$ performed $\sim 20 \%$ lower peak power across the 5-wk training (main effect of leg, $P=0.001$ ).

Endurance performance. Time to exhaustion in the onelegged incremental test increased 22\% $(P=0.001)$ after AE + RE and was unchanged after RE (interaction: $P=0.002$, Table 1 ). Peak HR was similar across time and leg (Table 1). At failure, RPE (local) amounted to $19 \pm 1$ for both legs at pre- and posttests.

Strength performance. Flywheel knee extension peak torque increased after training (Table 1). However, while peak ECC torque increased equally across legs, peak CON torque in- 


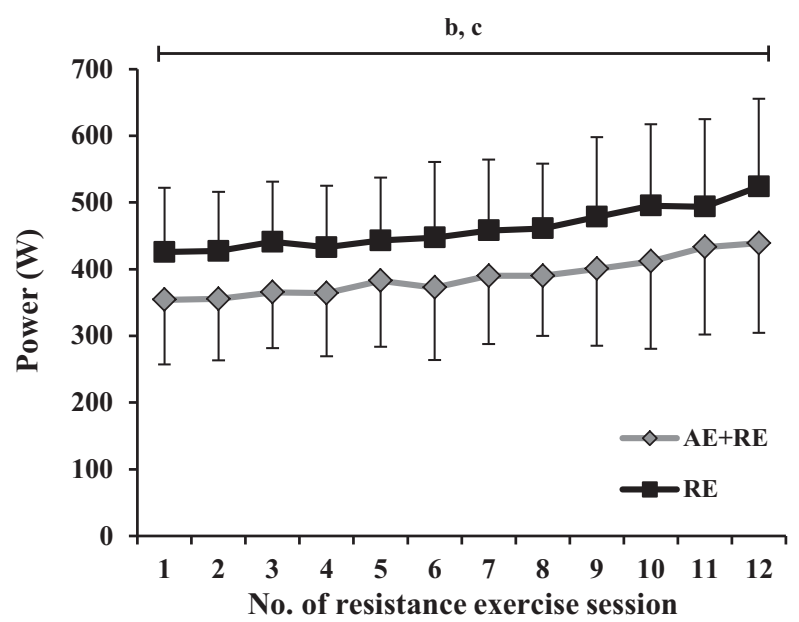

Fig. 2. Peak power measured in the flywheel ergometer during 12 resistance exercise sessions with $(\mathrm{AE}+\mathrm{RE})$ or without $(\mathrm{RE})$ preceding aerobic exercise. Means $\pm \mathrm{SD}$. Significant effect $(P<0.05): \mathrm{b}=1$ leg; $\mathrm{c}=$ time

creased after RE (10\%, $P=0.004)$, yet remained unchanged after $\mathrm{AE}+\mathrm{RE}(P=0.237)$. Consequently, $\mathrm{CON}$ torque normalized to muscle CSA was compromised after AE + RE training (interaction $P=0.010$ ). Maximal isometric strength was unaltered by training (Table 1). The isokinetic tests showed large variations in individual responses, resulting in no differences across time or legs (all $P>0.05$, Table 1 ).

Muscle volume, CSA, and signal intensity. Total QF volume and CSA increased after either intervention (Table 2). However, the increase was greater after $\mathrm{AE}+\mathrm{RE}(6 \%)$ than $\mathrm{RE}$ (3\%, Fig. 3; interaction QF volume: $F=38.5, P<0.0005$ ). Likewise, the increased volume of the four individual QF muscles was greater after $\mathrm{AE}+\mathrm{RE}$ than $\mathrm{RE}$ (Table 2). SI increased $7 \%$ after $\mathrm{AE}+\mathrm{RE}(P=0.009)$, yet was unaltered by
RE. This effect was consistent across the four individual QF muscles (Table 2). BF of either leg showed no change in SI.

Glycogen content. There was a leg $\times$ time interaction for glycogen content $(F=19.5, P=0.001)$. Thus at PRE, the leg that had completed AE showed $32 \%$ lower $(P<0.0005)$ glycogen concentration than the rested leg (Fig. 4). This effect was still evident $3 \mathrm{~h}$ after the acute RE bout $(P<0.0005)$. In the rested state after training, the leg subjected to $\mathrm{AE}+\mathrm{RE}$ showed greater glycogen content than the RE leg $(P=0.003)$ and compared with basal values $(P=0.004)$.

CS activity. CS activity increased $(18 \% ; P=0.001)$ in AE + $\mathrm{RE}$ (from 44.8 to $53.1 \mathrm{mmol} \cdot \mathrm{kg}^{-1} \cdot \mathrm{min}^{-1}$ ), but not in RE (43.3 $\left.\mathrm{mmol} \cdot \mathrm{kg}^{-1} \cdot \mathrm{min}^{-1}\right)$.

Gene expression. There was a leg $\times$ time interaction for PGC-1 $\alpha$ expression $(F=116.6, P<0.0005$; Fig. 5). Thus the increased expression from pre to post was greater after $\mathrm{AE}+$ RE (10.3-fold, $P<0.0005)$, than RE (2.0-fold, $P=0.001)$. Likewise, there was an interaction effect for VEGF expression $(F=28.4, P<0.0005)$, due to a greater increase after $\mathrm{AE}+$ RE (2.5-fold, $P<0.0005)$ vs. RE (1.2-fold, $P<0.042)$. Myostatin expression showed a tendency for interaction $(P=$ $0.086)$ because the downregulation was greater after $\mathrm{AE}+\mathrm{RE}$ $(65 \% ; P<0.0005)$ than RE (31\%; not significant after FDR procedures). MuRF-1 expression increased after AE + RE (2.9-fold, $P=0.003$ ) and was unchanged after RE (interaction: $F=20.4, P=0.001)$. Atrogin-1 showed interaction $(F=42.0$, $P<0.0005)$, as mRNA levels decreased after RE (43\%, $P=$ $0.004)$ and tended to increase after $\mathrm{AE}+\mathrm{RE}$ (1.3-fold, $P=$ $0.101)$.

Protein phosphorylation. AMPK phosphorylation showed a leg $\times$ time interaction $(F=11.4, P=0.008)$; values at PRE were greater $(1.5$-fold, $P=0.034)$ in the leg that had performed AE, compared with the "rested" (RE) leg (Fig. 6). Similarly, rpS6 signaling showed interaction $(F=14.4, P=$ $0.004)$ such that phosphorylation was elevated (1.3-fold, $P=$

Table 1. Selected performance measures pre and post resistance training with $(A E+R E)$ or without $(R E)$ preceding aerobic exercise

\begin{tabular}{|c|c|c|c|c|c|c|}
\hline & \multicolumn{3}{|c|}{$\mathrm{AE}+\mathrm{RE}$} & \multicolumn{3}{|c|}{$\mathrm{RE}$} \\
\hline Endurance performance $\mathrm{e}^{\mathrm{a}, \mathrm{b}, \mathrm{c}}, \mathrm{s}$ & $632 \pm 105$ & $770 \pm 145^{*} \#$ & 22 & $624 \pm 151$ & $654 \pm 142$ & 5 \\
\hline Peak heart rate at $\mathrm{W}_{\max }{ }^{\mathrm{b}}$, beats/min & $163 \pm 7$ & $168 \pm 16$ & 3 & $160 \pm 10$ & $159 \pm 15$ & -1 \\
\hline Flywheel merged peak power ${ }^{\mathrm{c}}, \mathrm{W}$ & $428 \pm 110$ & $496 \pm 129 *$ & 16 & $435 \pm 80$ & $515 \pm 109^{*}$ & 18 \\
\hline Flywheel peak CON powerc ${ }^{\mathrm{c}}$ W & $425 \pm 105$ & $480 \pm 126^{*}$ & 13 & $427 \pm 76$ & $501 \pm 104 *$ & 17 \\
\hline Flywheel peak ECC powerc, W & $432 \pm 116$ & $512 \pm 135^{*}$ & 19 & $443 \pm 88$ & $529 \pm 118^{*}$ & 19 \\
\hline Flywheel peak CON torque ${ }^{\mathrm{a}, \mathrm{c}}, \mathrm{N} \cdot \mathrm{m}$ & $236 \pm 33$ & $247 \pm 43$ & 5 & $234 \pm 26$ & $258 \pm 35^{*}$ & 10 \\
\hline Normalized CON torque $^{\mathrm{a}}, \mathrm{N} \cdot \mathrm{m} / \mathrm{cm}^{2}$ & $2.95 \pm 0.28$ & $2.90 \pm 0.34 \#$ & -2 & $2.87 \pm 0.26$ & $3.08 \pm 0.39 *$ & 7 \\
\hline Flywheel peak ECC torque ${ }^{\mathrm{c}}, \mathrm{N} \cdot \mathrm{m}$ & $263 \pm 44$ & $301 \pm 63^{*}$ & 14 & $268 \pm 46$ & $303 \pm 51 *$ & 13 \\
\hline Normalized ECC torque, $\mathrm{N} \cdot \mathrm{m} / \mathrm{cm}^{2}$ & $3.29 \pm 0.55$ & $3.56 \pm 0.66 *$ & 8 & $3.29 \pm 0.54$ & $3.64 \pm 0.71 *$ & 11 \\
\hline Maximal isometric torque, $\mathrm{N} \cdot \mathrm{m}$ & $323 \pm 84$ & $305 \pm 52$ & -6 & $297 \pm 52$ & $314 \pm 68$ & 6 \\
\hline \multicolumn{7}{|l|}{ Isometric torque, $\mathrm{N} \cdot \mathrm{m}$} \\
\hline at $4.19 \mathrm{rad} / \mathrm{s}$ & $140 \pm 35$ & $128 \pm 31$ & -9 & $140 \pm 26$ & $141 \pm 37$ & 1 \\
\hline
\end{tabular}

Values are means \pm SD. $\mathrm{W}_{\text {max }}$, maximal workload; CON, concentric; ECC, eccentric. Significant main effects $(P<0.05): \mathrm{a}=$ interaction, $\mathrm{b}=$ leg, $\mathrm{c}=$ time. Significant simple effects $(P<0.05)$ : *time (POST vs. PRE); \#leg (AE + RE vs. RE). 
Table 2. Selected MRI measures pre and post resistance training with $(A E+R E)$ or without $(R E)$ preceding aerobic exercise

\begin{tabular}{|c|c|c|c|c|c|c|}
\hline & \multicolumn{3}{|c|}{$\mathrm{AE}+\mathrm{RE}$} & \multicolumn{3}{|c|}{$\mathrm{RE}$} \\
\hline QF muscle volume ${ }^{\mathrm{a}, \mathrm{c}}, \mathrm{cm}^{3}$ & $1,217 \pm 246$ & $1,286 \pm 247 *$ & 6 & $1,239 \pm 231$ & $1,273 \pm 237 *$ & 3 \\
\hline VI muscle volume $\mathrm{a}^{\mathrm{a}, \mathrm{c}}, \mathrm{cm}^{3}$ & $376 \pm 77$ & $389 \pm 79 *$ & 3 & $390 \pm 67$ & $395 \pm 71$ & 1 \\
\hline VM muscle volume $e^{a, c}, \mathrm{~cm}^{3}$ & $322 \pm 70$ & $336 \pm 69^{*} \#$ & 4 & $318 \pm 64$ & $325 \pm 64^{*}$ & 2 \\
\hline $\mathrm{RF}$ muscle volume $\mathrm{a}^{\mathrm{a}, \mathrm{c}}, \mathrm{cm}^{3}$ & $117 \pm 26$ & $135 \pm 28 *$ & 15 & $120 \pm 33$ & $131 \pm 34^{*}$ & 9 \\
\hline $\mathrm{QF}$ mean $\mathrm{CSA}^{\mathrm{a}, \mathrm{c}}, \mathrm{cm}^{2}$ & $80.7 \pm 13.2$ & $85.2 \pm 12.9^{*}$ & 6 & $82.2 \pm 12.5$ & $84.4 \pm 12.5^{*}$ & 3 \\
\hline VI signal intensity ${ }^{\mathrm{a}}, \mathrm{MGV}$ & $47.4 \pm 4.3$ & $49.8 \pm 4.8^{* \#}$ & 5 & $46.5 \pm 4.6$ & $45.5 \pm 4.4$ & -2 \\
\hline VM signal intensity ${ }^{\mathrm{a}}, \mathrm{MGV}$ & $50.4 \pm 5.9$ & $51.9 \pm 7.0$ & 3 & $49.6 \pm 7.4$ & $48.2 \pm 7.3$ & -3 \\
\hline RF signal intensity ${ }^{\mathrm{a}, \mathrm{c}}, \mathrm{MGV}$ & $47.0 \pm 11.9$ & $53.2 \pm 15.4^{*}$ & 13 & $46.6 \pm 9.5$ & $46.8 \pm 10.5$ & 0 \\
\hline BF signal intensity, MGV & $41.2 \pm 5.7$ & $41.6 \pm 5.8$ & 1 & $39.7 \pm 4.2$ & $39.9 \pm 5.1$ & 1 \\
\hline
\end{tabular}

Values are means \pm SD. CSA, cross-sectional area; MGV, mean gray value; QF, quadriceps femoris; VL, vastus lateralis; VI, vastus intermedius; VM, vastus medialis; RF, rectus femoris; BF, biceps femoris. Significant main effects $(P<0.05)$ : a $=$ interaction, $\mathrm{b}=$ leg, $\mathrm{c}=$ time. Significant simple effects $(P<0.05)$ : *time (PRE vs. POST); \#leg (AE + RE vs. RE).

0.021) in $\mathrm{AE}+\mathrm{RE}$ at PRE. Although 4E-BP1 signaling was similar across legs, there was a trend toward a main effect of time $(P=0.070)$ due to a slight increase from PRE to POST. There were no changes across time or legs for p70S6K phosphorylation. Representative blots are shown in Fig. 7.

\section{DISCUSSION}

The current study scrutinized the proposed negative effect of AE-induced AMPK activation on subsequent muscle signaling and hypertrophic responses to RE training. Our novel results show that AMPK activation prompted by AE does not compromise hypertrophy in human muscle subjected to RE. Indeed, concurrent $\mathrm{AE}+\mathrm{RE}$ rather produced greater increase in muscle size than RE. Thus, while we refute the purported incompatibility between AMPK signaling and muscle hypertrophic responses, the results reinforce that $\mathrm{AE}$ could obstruct the progression of in vivo muscle function to subsequent cumulative RE. If employed in competitive athletes or executed over extended time, there are obvious reasons to suspect this effect would have adverse impact on performance.

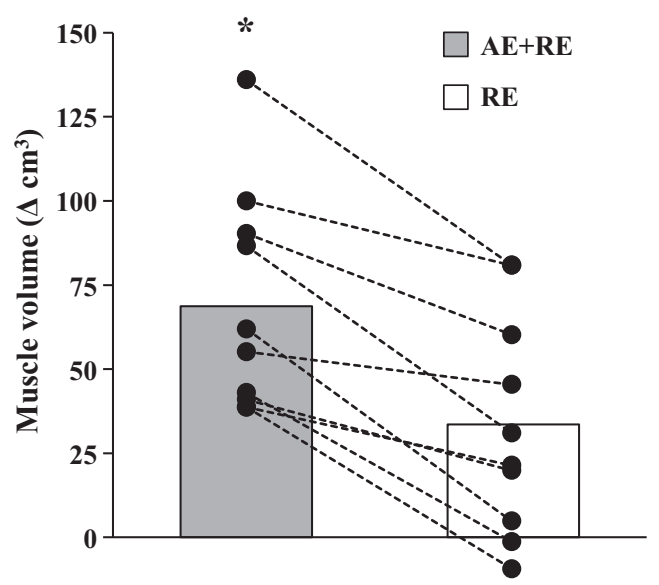

Fig. 3. Individual and mean increase in quadriceps muscle volume after resistance training with $(\mathrm{AE}+\mathrm{RE})$ or without $(\mathrm{RE})$ concurrent aerobic exercise. ${ }^{*}$ Greater increase after $\mathrm{AE}+\mathrm{RE}$.
AMPK activation induced by AE has been held responsible for interfering with muscle growth to subsequent RE training $(4,27)$. Indeed, animal and in vitro studies suggest AMPK signaling blocks mTOR activity (34), impairs protein synthesis $(8,60)$, and provokes myofibrillar protein degradation (51). In stark contrast, and somewhat as a surprise, we recently reported robust muscle hypertrophy after RE preceded by $\mathrm{AE}$, and allowing for $6 \mathrm{~h}$ recovery, that overshadowed that produced by RE only (42). Collectively these findings spurred us to hypothesize that recovery between exercise bouts is a prerequisite to optimize functional and cellular responses to concurrent $\mathrm{AE}+\mathrm{RE}$ training.

In the current investigation, and similar to the findings of our previous study (42), AE + RE produced more substantial increases in muscle size than RE. Thus inevitably, back-toback AE + RE did not induce AMPK-mediated blunting of muscle hypertrophy. This may be because suppressed protein synthesis is associated with increased AMPK signaling only during and immediately after acute exercise (20). In fact, there

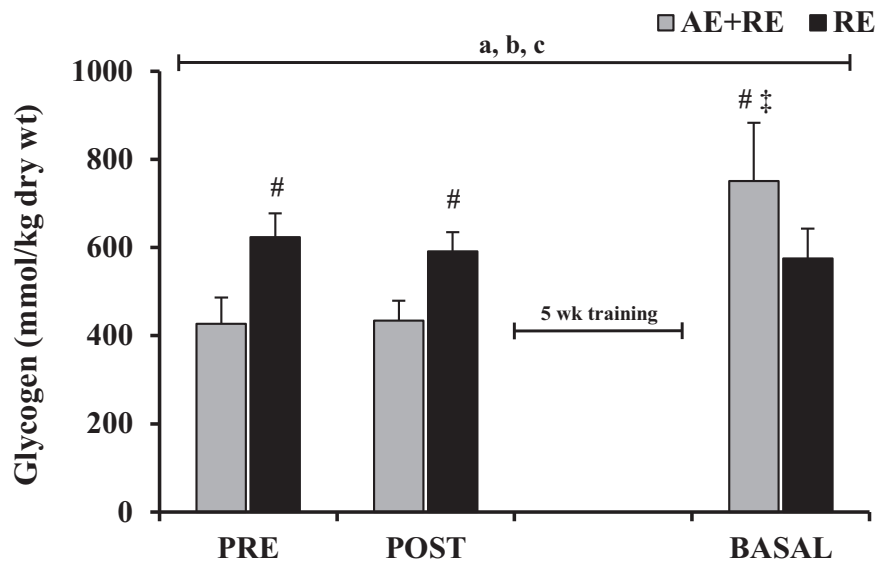

Fig. 4. Muscle glycogen concentration before (PRE) and $3 \mathrm{~h}$ after (POST) acute resistance exercise with $(\mathrm{AE}+\mathrm{RE})$ or without $(\mathrm{RE})$ preceding aerobic exercise and in the rested state (BASAL) after $5 \mathrm{wk}$ training. Significant effect $(P<0.05): \mathrm{a}=$ interaction, $\mathrm{b}=1 \mathrm{eg}, \mathrm{c}=$ time. Significant differences $(P<$ $0.05)$ vs. \#opposite leg. 
Fig. 5. A-E: PGC-1 $\alpha$, VEGF, MuRF-1, atrogin-1, and myostatin mRNA levels before (PRE) and $3 \mathrm{~h}$ after (POST) resistance exercise with $(\mathrm{AE}+\mathrm{RE})$ or without $(\mathrm{RE})$ preceding aerobic exercise. Significant effects $(P<0.05)$ : $\mathrm{a}=$ interaction, $\mathrm{b}=1$ leg, $\mathrm{c}=$ time. Significant differences $(P<0.05)$ vs. \#opposite leg, *same leg at PRE.
A

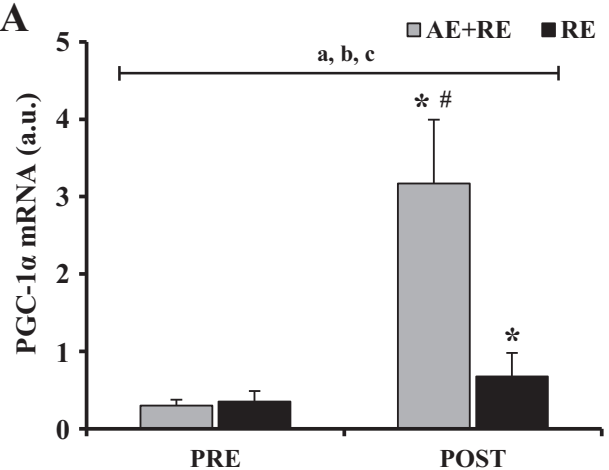

C

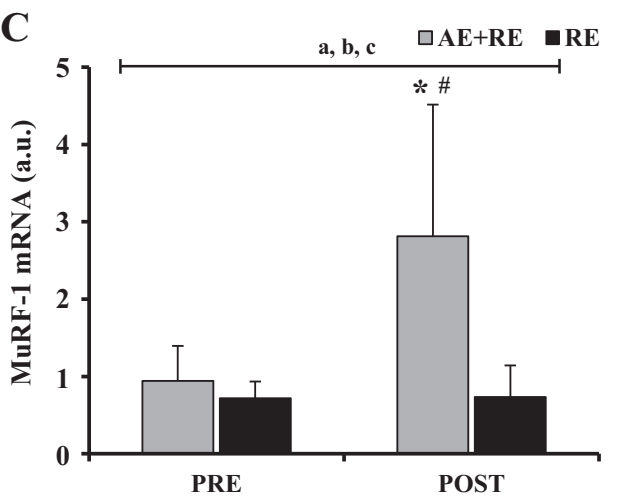

$\mathbf{E}$

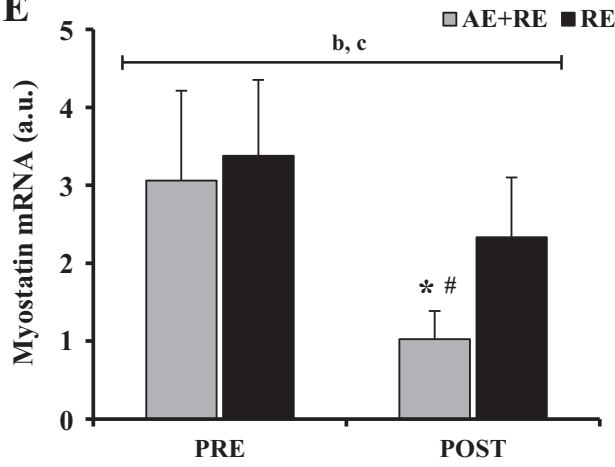

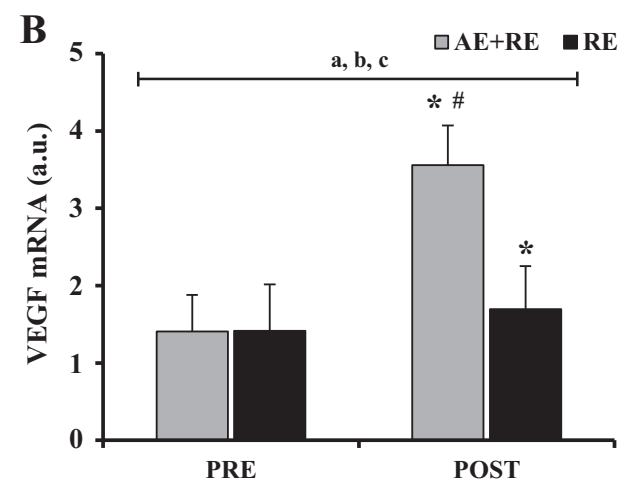

D

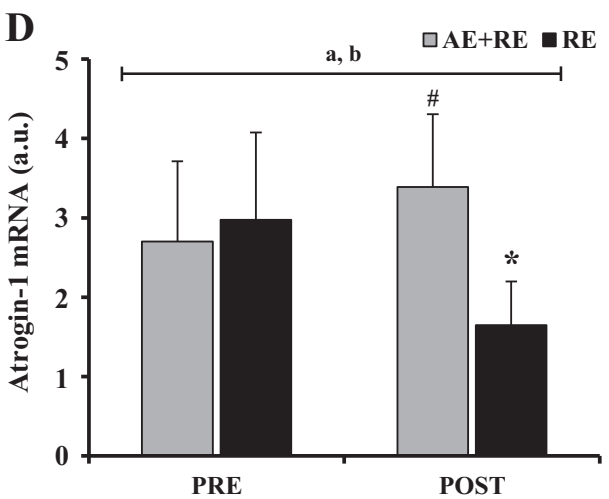

is sound support for this presumption. First, increased AMPK activity is typically evident $\leq 1 \mathrm{~h}$ postexercise $(11,20,46)$ returning to baseline shortly thereafter $(20,62)$, and cumulative exercise may even offset this response (46). Second, when RE was executed subsequent to $\mathrm{AE}$ (15), the only inhibitory effect occurred at the IGF-1 mRNA level while mTOR signaling was not impacted. Third, muscle protein fractional synthetic rate, assessed 1-4 $\mathrm{h}$ postexercise, was comparable across $\mathrm{AE}+\mathrm{RE}$ and RE (13). Taken together, and given that protein synthesis may be elevated $48-72 \mathrm{~h}$ after acute RE (38), the very short-lived AMPK activation induced by $\mathrm{AE}$ most likely evokes minute, if any, impact on the net protein balance accumulated between exercise sessions.

While the lack of enhanced mTOR signaling after RE was unexpected, rpS6 phosphorylation was elevated after AE. This increase occurred in parallel with increased AMPK phosphorylation following $\mathrm{AE}$, yet was unaccompanied by increases in other mTOR effectors. This response seems at odds with the current understanding of enhanced translation initiation and protein synthesis after acute exercise. However, increases in muscle protein synthesis and rpS6 phosphorylation may occur in the absence of increased mTOR signaling (19). Further, our gene expression records support that $\mathrm{AE}+\mathrm{RE}$ served a more potent stimulus for tissue remodeling than RE. More specifically, while RE alone failed to downregulate myostatin and increase MuRF-1 expression, these genes showed robust responses after AE + RE. Myostatin suppression has been associated with muscle hypertrophy and decreased specific force (47), and MuRF-1 expression appears crucial for loadinginduced growth to occur (33). These findings largely also corroborate with changes in expression of other genes (i.e., PGC-1 $\alpha$, VEGF and atrogin-1), which showed augmented mRNA response in the leg that was subjected to concurrent exercise. These results are intriguing, because apart from controlling mitochondrial biogenesis induced by AE, PGC- $1 \alpha$ induction may also protect against muscle protein breakdown (10) and elicit hypertrophy in response to loading (57). Further, it is noteworthy our results accord with the exaggerated gene expression response to $\mathrm{AE}+\mathrm{RE}$ with $6 \mathrm{~h}$ between bouts (22, $41)$. Thus, at least during the initial training phase and regard- 
A

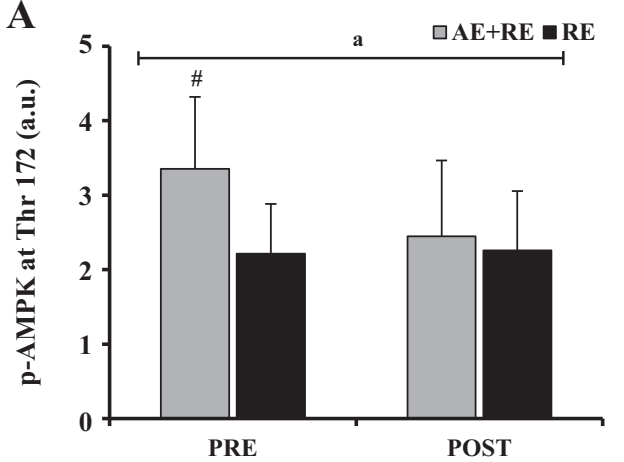

C

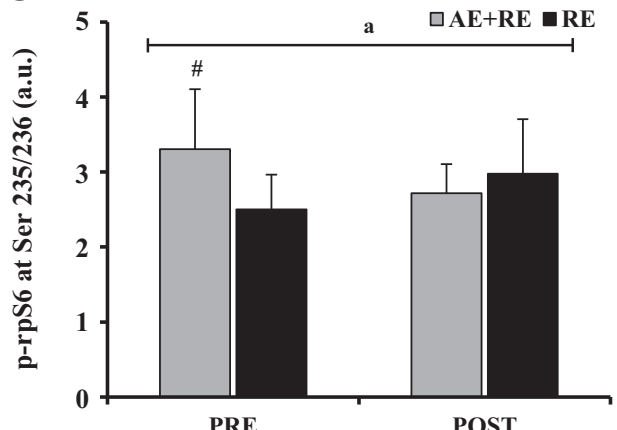

B

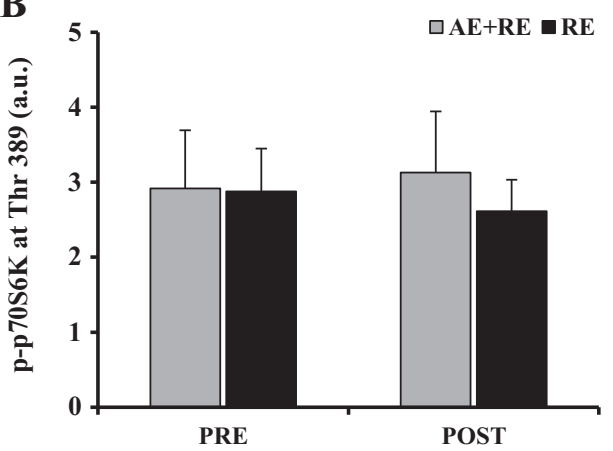

D

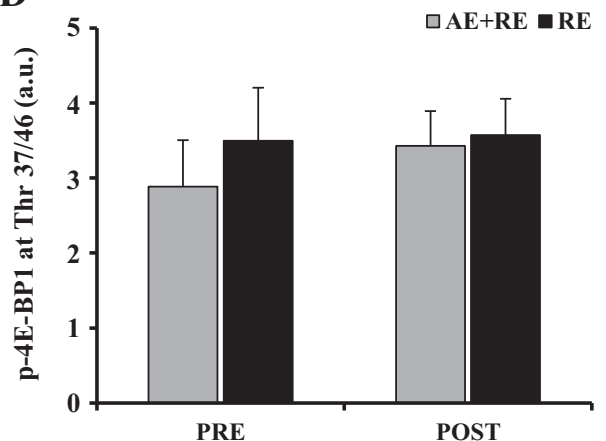

Fig. 6. $A-D$ : phosphorylation of phosphoP70S6K (Thr389), phospho-rpS6 (Ser235/ 236), phospho-4E-BP1 (Thr37/46), and phosphoAMPK $\alpha($ Thr172) relative to total $\alpha$-tubulin before (PRE) and $3 \mathrm{~h}$ after (POST) resistance exercise with $(\mathrm{AE}+\mathrm{RE})$ or without (RE) preceding aerobic exercise. Significant effects $(P<0.05)$ : a $=$ interaction. Significant differences $(P<0.05)$ vs. \#opposite leg. less of recovery, it appears the more voluminous $\mathrm{AE}+\mathrm{RE}$ paradigm induced a more favorable cellular environment for muscular, vascular, and mitochondrial protein turnover.

It is well established that AE reduces glycogen stores, and commencing exercise in a low-glycogen state may evoke different metabolic and molecular responses compared with muscles displaying normal or supranormal glycogen levels (17, $28)$. It has been postulated that glycogen depletion, and/or associated changes in muscle energy balance, could contribute to the incompatibility of mixed-mode training $(40,50)$. In the current study, however, glycogen utilization induced by $\mathrm{AE}$ was not accompanied by altered mTOR signaling $3 \mathrm{~h}$ post RE.

$$
\frac{\mathrm{RE}}{\text { Pre Post }} \frac{\mathrm{AE}+\mathrm{RE}}{\text { Pre Post }}
$$

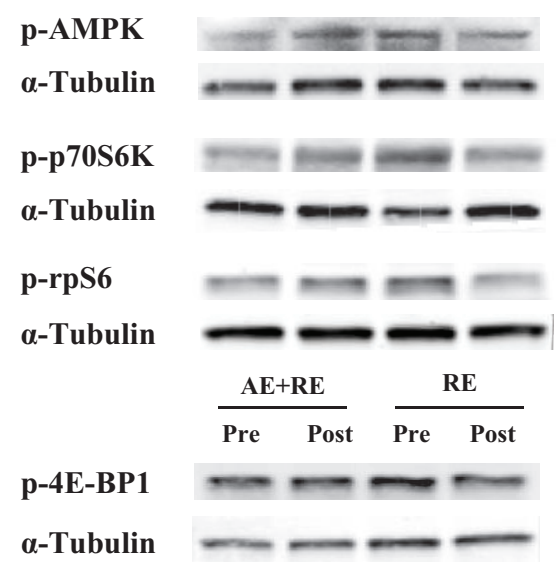

Fig. 7. Representative blots of phospho-P70S6K (Thr389), phospho-rpS6 (Ser235/236), phospho-4E-BP1 (Thr37/46) and phospho-AMPK $\alpha$ (Thr172) with corresponding $\alpha$-tubulin bands.
Similarly, translational signaling and protein synthetic responses to RE were not compromised in glycogen-voided skeletal muscle at onset of exercise (12). Taken together, it appears human muscle commencing RE at $\sim 30 \%$ reduced glycogen stores and $\sim 20 \%$ compromised function possesses undiminished ability to undergo hypertrophy.

Evidently $\mathrm{AE}+\mathrm{RE}$ exaggerated the increased muscle size shown with RE only. These findings were very consistent and the hierarchical response across individual QF muscles was identical to what we have reported earlier $(42,52)$. Moreover, $\mathrm{SI}$ of the RE leg and the nonused BF muscle serving as control was unaltered, providing further evidence that reported differences across legs were specific to the QF muscle group and the intervention imposed. However, given the established relationship between muscle anatomical CSA and in vivo force (44), the finding of greater muscle hypertrophy, but not force, after $\mathrm{AE}+\mathrm{RE}$ than RE suggests that the increase in size was not entirely "functional." At first, and consistent with the notion of increased SI of MRIs $(26,42)$, it would be tempting to attribute the reduced specific force to edema or swelling due to the preceding exercise. However, it should be recalled both legs executed the high-load ECC component of RE, and none of the subjects reported delayed onset of muscle soreness, associated with ECC exercise (23). It also remains that the acute molecular response implied greater anabolic environment after $\mathrm{AE}+$ RE compared with RE. Likewise, muscle samples obtained before and after $5 \mathrm{wk}$ AE + RE or RE (42) showed unaltered protein concentrations despite the robust hypertrophy (unpublished observation). Altogether, these findings suggest that the increased SI was caused by events unrelated to cell swelling. As mitochondria constitute $4-6 \%$ of muscle tissue (32) and our proxy markers (CS activity; endurance performance) showed substantial increases after $\mathrm{AE}+\mathrm{RE}$, it may be that 
noncontractile protein constituents had subtle, yet significant impact on size measures. In fact, increases in mitochondrial volume and glycogen stores after short-term endurance training have been estimated to account for 3-4\% of the increase in muscle CSA $(53,54)$. While it remains to establish a credible explanation for the more ample increase in muscle volume after $\mathrm{AE}+\mathrm{RE}$ than RE, evidently increases in both myofibrillar and mitochondrial/sarcoplasmic protein pools occur in parallel.

Albeit the AE insult impaired succeeding RE performance throughout the training period (Fig. 2), force and power increased after both $\mathrm{AE}+\mathrm{RE}$ and $\mathrm{RE}$ in the current investigation. However, RE-induced improvements in normalized and absolute CON torque were blunted by $\mathrm{AE}+\mathrm{RE}$. Previous research also suggests that concurrent training may interfere with the progression of in vivo muscle function (40). Such interference appears most evident for explosive strength and power $(21,24,48,55)$ and is not necessarily accompanied by compromised muscle hypertrophy $(25,35,48)$. Regardless of mechanism(s) involved, it is imperative that certain unidentified events responsible for functional shortcomings brought about by the previous $\mathrm{AE}$, must be normalized prior to RE. In support, high- but not low-intensity RE produced strength gains, whereas muscle size increased regardless of intensity (31). Further, back-to-back AE + RE impeded progression of strength, but not muscle size (58). Concurrent exercise allowing for $6 \mathrm{~h}$ recovery between exercise modes did not (42). Altogether, it is apparent that restored muscle function between exercise bouts is a prerequisite for attaining optimal gains in muscle function in response to $\mathrm{AE}+\mathrm{RE}$ training. This contrasts the muscle hypertrophic response, which seems to occur independent of recovery.

The one-legged AE model employed here promotes early endurance adaptations $(42,49,56)$. This was manifested in marked increases in PGC- $1 \alpha$ and VEGF expression after acute $\mathrm{AE}$, and enhanced CS activity and endurance performance after $\mathrm{AE}+\mathrm{RE}$ training. The current RE protocol increased muscle size and strength at rates comparable to what has been shown elsewhere $(52,61)$. While we have no apparent explanation for the somewhat less pronounced RE-induced increases in muscle size and strength than reported by us recently using the same RE paradigm (42), this observation should not distract from the mutual finding of significantly different response across $\mathrm{AE}+\mathrm{RE}$ and RE in the two studies.

It should be acknowledged that in the current study, total work performed differed across legs. Such a response is inherent in any experimental design aimed at examining the effect of $\mathrm{AE}$ on responses prompted by RE. Given the particular question posted here, this effect presents no drawback in interpreting our results. The unique loading feature of the RE methodology used here allows for execution of maximal voluntary force or power through the entire range of motion in each CON action of each set, as well as ECC overload (59). Inevitably, the preceding $\mathrm{AE}$, which consisted of $\mathrm{CON}$ knee extensions only, compromised peak power during this task (Fig. 2). In this context it should be appreciated that employing an AE mode other than the current cycling model, e.g., running comprising ECC actions and characterized by different loading history, may have produced different results. While being outside the scope of the present study, a recent meta-analysis, quantifying a total of 422 effect sizes, reported that the interference effect is exacerbated with running compared with cycling (63).

In conclusion, consecutive bouts of $\mathrm{AE}+\mathrm{RE}$ performed over 5 wk exaggerated the increase in muscle size shown with RE alone. This occurred despite increased AMPK phosphorylation, reduced glycogen content, and attenuated muscle function elicited by AE. Thus we demonstrate that AMPK activation induced by $\mathrm{AE}$ does not interfere with muscle growth produced by concurrent RE training. However, AE + RE blunt the progression of important aspects of in vivo muscle function. Employing this approach may therefore be counterproductive for athletes and individuals aiming at developing maximal strength and power.

\section{ACKNOWLEDGMENTS}

We thank D. Carlsson, R. Vargas Paris, and M. Pettersson for technical support.

\section{GRANTS}

This study was supported by grants from the Swedish National Centre for Research in Sports (P. A. Tesch; CIF), the European Space Agency (P. A. Tesch; ESA), and the Swedish National Space Board (P. A. Tesch; SNSB).

\section{DISCLOSURES}

No conflicts of interest, financial or otherwise, are declared by the author(s).

\section{AUTHOR CONTRIBUTIONS}

Author contributions: T.R.L., R.F.-G., and P.A.T. conception and design of research; T.R.L. and R.F.-G. performed experiments; T.R.L. and R.F.-G. analyzed data; T.R.L., R.F.-G., and P.A.T. interpreted results of experiments; T.R.L. and P.A.T. drafted manuscript; T.R.L., R.F.-G., and P.A.T. edited and revised manuscript; T.R.L., R.F.-G., and P.A.T. approved final version of manuscript; R.F.-G. prepared figures.

\section{REFERENCES}

1. Andersen P, Adams RP, Sjogaard G, Thorboe A, Saltin B. Dynamic knee extension as model for study of isolated exercising muscle in humans. J Appl Physiol 59: 1647-1653, 1985.

2. Apro W, Wang L, Ponten M, Blomstrand E, Sahlin K. Resistance exercise induced mTORC1 signalling is not impaired by subsequent endurance exercise in human skeletal muscle. Am J Physiol Endocrinol Metab 305: E22-E32, 2013.

3. Atherton PJ, Babraj J, Smith K, Singh J, Rennie MJ, Wackerhage H. Selective activation of AMPK-PGC-1alpha or PKB-TSC2-mTOR signaling can explain specific adaptive responses to endurance or resistance training-like electrical muscle stimulation. FASEB J 19: 786-788, 2005.

4. Baar K. Training for endurance and strength: lessons from cell signaling. Med Sci Sports Exerc 38: 1939-1944, 2006.

5. Bentley DJ, Smith PA, Davie AJ, Zhou S. Muscle activation of the knee extensors following high intensity endurance exercise in cyclists. Eur $J$ Appl Physiol 81: 297-302, 2000.

6. Berg HE, Tedner B, Tesch PA. Changes in lower limb muscle crosssectional area and tissue fluid volume after transition from standing to supine. Acta Physiol Scand 148: 379-385, 1993.

7. Bergström J. Muscle electrolytes in man. Scand J Clin Lab Invest 14: $1-110,1962$.

8. Bolster DR, Crozier SJ, Kimball SR, Jefferson LS. AMP-activated protein kinase suppresses protein synthesis in rat skeletal muscle through down-regulated mammalian target of rapamycin (mTOR) signaling. $J$ Biol Chem 277: 23977-23980, 2002.

9. Borg GA. Psychophysical bases of perceived exertion. Med Sci Sports Exerc 14: 377-381, 1982.

10. Brault JJ, Jespersen JG, Goldberg AL. Peroxisome proliferator-activated receptor gamma coactivator 1alpha or 1beta overexpression inhibits muscle protein degradation, induction of ubiquitin ligases, and disuse atrophy. J Biol Chem 285: 19460-19471, 2010. 
11. Camera DM, Edge J, Short MJ, Hawley JA, Coffey VG. Early time course of Akt phosphorylation after endurance and resistance exercise. Med Sci Sports Exerc 42: 1843-1852, 2010.

12. Camera DM, West DW, Burd NA, Phillips SM, Garnham AP, Hawley JA, Coffey VG. Low muscle glycogen concentration does not suppress the anabolic response to resistance exercise. J Appl Physiol 113: 206-214, 2012

13. Carrithers JA, Carroll CC, Coker RH, Sullivan DH, Trappe TA. Concurrent exercise and muscle protein synthesis: implications for exercise countermeasures in space. Aviat Space Environ Med 78: 457-462, 2007.

14. Coffey VG, Hawley JA. The molecular bases of training adaptation. Sports Med 37: 737-763, 2007.

15. Coffey VG, Pilegaard H, Garnham AP, O'Brien BJ, Hawley JA. Consecutive bouts of diverse contractile activity alter acute responses in human skeletal muscle. J Appl Physiol 106: 1187-1197, 2009.

16. Coffey VG, Zhong Z, Shield A, Canny BJ, Chibalin AV, Zierath JR, Hawley JA. Early signaling responses to divergent exercise stimuli in skeletal muscle from well-trained humans. FASEB J 20: 190-192, 2006.

17. Creer A, Gallagher P, Slivka D, Jemiolo B, Fink W, Trappe S. Influence of muscle glycogen availability on ERK1/2 and Akt signaling after resistance exercise in human skeletal muscle. J Appl Physiol 99: 950-956, 2005.

18. Curran-Everett D. Multiple comparisons: philosophies and illustrations. Am J Physiol Regul Integr Comp Physiol 279: R1-R8, 2000.

19. Donges CE, Burd NA, Duffield R, Smith GC, West DW, Short MJ, Mackenzie R, Plank LD, Shepherd PR, Phillips SM, Edge JA. Concurrent resistance and aerobic exercise stimulates both myofibrillar and mitochondrial protein synthesis in sedentary middle-aged men. $J$ Appl Physiol 112: 1992-2001, 2012.

20. Dreyer HC, Fujita S, Cadenas JG, Chinkes DL, Volpi E, Rasmussen BB. Resistance exercise increases AMPK activity and reduces 4E-BP1 phosphorylation and protein synthesis in human skeletal muscle. $J$ Physiol 576: 613-624, 2006

21. Dudley GA, Djamil R. Incompatibility of endurance- and strengthtraining modes of exercise. J Appl Physiol 59: 1446-1451, 1985.

22. Fernandez-Gonzalo R, Lundberg TR, Tesch PA. Acute molecular responses in untrained and trained muscle subjected to aerobic and resistance exercise training versus resistance training alone. Acta Physiol (Oxf) 209: 283-294, 2013.

23. Foley JM, Jayaraman RC, Prior BM, Pivarnik JM, Meyer RA. MR measurements of muscle damage and adaptation after eccentric exercise. $J$ Appl Physiol 87: 2311-2318, 1999.

24. Glowacki SP, Martin SE, Maurer A, Baek W, Green JS, Crouse SF. Effects of resistance, endurance, and concurrent exercise on training outcomes in men. Med Sci Sports Exerc 36: 2119-2127, 2004.

25. Hakkinen $\mathbf{K}$, Alen $\mathbf{M}$, Kraemer WJ, Gorostiaga $\mathbf{E}$, Izquierdo $\mathbf{M}$, Rusko H, Mikkola J, Hakkinen A, Valkeinen H, Kaarakainen E, Romu S, Erola V, Ahtiainen J, Paavolainen L. Neuromuscular adaptations during concurrent strength and endurance training versus strength training. Eur J Appl Physiol 89: 42-52, 2003.

26. Harber MP, Konopka AR, Douglass MD, Minchev K, Kaminsky LA, Trappe TA, Trappe S. Aerobic exercise training improves whole muscle and single myofiber size and function in older women. Am J Physiol Regul Integr Comp Physiol 297: R1452-R1459, 2009.

27. Hawley JA. Molecular responses to strength and endurance training: are they incompatible? Appl Physiol Nutr Metab 34: 355-361, 2009.

28. Hawley JA, Burke LM, Phillips SM, Spriet LL. Nutritional modulation of training-induced skeletal muscle adaptations. J Appl Physiol 110: $834-845,2011$.

29. Hermansen L, Hultman E, Saltin B. Muscle glycogen during prolonged severe exercise. Acta Physiol Scand 71: 129-139, 1967.

30. Hickson RC. Interference of strength development by simultaneously training for strength and endurance. Eur J Appl Physiol Occup Physiol 45: 255-263, 1980.

31. Holm L, Reitelseder S, Pedersen TG, Doessing S, Petersen SG, Flyvbjerg A, Andersen JL, Aagaard P, Kjaer M. Changes in muscle size and MHC composition in response to resistance exercise with heavy and light loading intensity. J Appl Physiol 105: 1454-1461, 2008.

32. Hoppeler H, Howald H, Conley K, Lindstedt SL, Claassen H, Vock P, Weibel ER. Endurance training in humans: aerobic capacity and structure of skeletal muscle. J Appl Physiol 59: 320-327, 1985.
33. Hwee DT, Baehr LM, Philp A, Baar K, Bodine SC. Maintenance of muscle mass and load-induced growth in Muscle RING Finger 1 null mice with age. Aging Cell 13: 92-101, 2014.

34. Inoki K, Zhu T, Guan KL. TSC2 mediates cellular energy response to control cell growth and survival. Cell 115: 577-590, 2003.

35. Izquierdo M, Hakkinen K, Ibanez J, Kraemer WJ, Gorostiaga EM. Effects of combined resistance and cardiovascular training on strength, power, muscle cross-sectional area, and endurance markers in middle-aged men. Eur J Appl Physiol 94: 70-75, 2005.

36. Jacobs I, Kaiser $\mathbf{P}$, Tesch $\mathbf{P}$. Muscle strength and fatigue after selective glycogen depletion in human skeletal muscle fibers. Eur J Appl Physiol Occup Physiol 46: 47-53, 1981.

37. Kraemer WJ, Patton JF, Gordon SE, Harman EA, Deschenes MR, Reynolds K, Newton RU, Triplett NT, Dziados JE. Compatibility of high-intensity strength and endurance training on hormonal and skeletal muscle adaptations. J Appl Physiol 78: 976-989, 1995.

38. Kumar V, Atherton P, Smith K, Rennie MJ. Human muscle protein synthesis and breakdown during and after exercise. J Appl Physiol 106: 2026-2039, 2009.

39. Leveritt M, Abernethy PJ. Acute effects of high-intensity endurance exercise on subsequent resistance activity. J Strength Cond Res 13: 47-51, 1999.

40. Leveritt M, Abernethy PJ, Barry BK, Logan PA. Concurrent strength and endurance training. A review. Sports Med 28: 413-427, 1999.

41. Lundberg TR, Fernandez-Gonzalo R, Gustafsson T, Tesch PA. Aerobic exercise alters skeletal muscle molecular responses to resistance exercise. Med Sci Sports Exerc 44: 1680-1688, 2012.

42. Lundberg TR, Fernandez-Gonzalo R, Gustafsson T, Tesch PA. Aerobic exercise does not compromise muscle hypertrophy response to short-term resistance training. J Appl Physiol 114: 81-89, 2013.

43. Mascher H, Andersson H, Nilsson PA, Ekblom B, Blomstrand E. Changes in signalling pathways regulating protein synthesis in human muscle in the recovery period after endurance exercise. Acta Physiol (Oxf) 191: 67-75, 2007.

44. Maughan RJ, Watson JS, Weir J. Strength and cross-sectional area of human skeletal muscle. J Physiol 338: 37-49, 1983.

45. Mayhew DL, Kim JS, Cross JM, Ferrando AA, Bamman MM. Translational signaling responses preceding resistance training-mediated myofiber hypertrophy in young and old humans. J Appl Physiol 107: $1655-1662,2009$.

46. McConell GK, Lee-Young RS, Chen ZP, Stepto NK, Huynh NN, Stephens TJ, Canny BJ, Kemp BE. Short-term exercise training in humans reduces AMPK signalling during prolonged exercise independent of muscle glycogen. J Physiol 568: 665-676, 2005.

47. Mendias CL, Kayupov E, Bradley JR, Brooks SV, Claflin DR. Decreased specific force and power production of muscle fibers from myostatin-deficient mice are associated with a suppression of protein degradation. J Appl Physiol 111: 185-191, 2011.

48. Mikkola J, Rusko H, Izquierdo M, Gorostiaga EM, Hakkinen K. Neuromuscular and cardiovascular adaptations during concurrent strength and endurance training in untrained men. Int J Sports Med 33: 702-710, 2012.

49. Mourtzakis M, Gonzalez-Alonso J, Graham TE, Saltin B. Hemodynamics and $\mathrm{O} 2$ uptake during maximal knee extensor exercise in untrained and trained human quadriceps muscle: effects of hyperoxia. $J$ Appl Physiol 97: 1796-1802, 2004.

50. Nader GA. Concurrent strength and endurance training: from molecules to man. Med Sci Sports Exerc 38: 1965-1970, 2006.

51. Nakashima K, Yakabe Y. AMPK activation stimulates myofibrillar protein degradation and expression of atrophy-related ubiquitin ligases by increasing FOXO transcription factors in $\mathrm{C} 2 \mathrm{C} 12$ myotubes. Biosci Biotechnol Biochem 71: 1650-1656, 2007.

52. Norrbrand L, Fluckey JD, Pozzo M, Tesch PA. Resistance training using eccentric overload induces early adaptations in skeletal muscle size. Eur J Appl Physiol 102: 271-281, 2008.

53. Nygren AT, Karlsson M, Norman B, Kaijser L. Effect of glycogen loading on skeletal muscle cross-sectional area and T2 relaxation time. Acta Physiol Scand 173: 385-390, 2001.

54. Nygren AT, Sundberg CJ, Goransson H, Esbjornsson-Liljedahl M, Jansson E, Kaijser L. Effects of dynamic ischaemic training on human skeletal muscle dimensions. Eur J Appl Physiol 82: 137-141, 2000.

55. Oliveira Ade S, Caputo F, Goncalves M, Denadai BS. Heavy-intensity aerobic exercise affects the isokinetic torque and functional but not 
conventional hamstrings:quadriceps ratios. J Electromyogr Kinesiol 19: 1079-1084, 2009.

56. Pilegaard H, Saltin B, Neufer PD. Exercise induces transient transcriptional activation of the PGC-1alpha gene in human skeletal muscle. $J$ Physiol 546: 851-858, 2003.

57. Ruas JL, White JP, Rao RR, Kleiner S, Brannan KT, Harrison BC, Greene NP, Wu J, Estall JL, Irving BA, Lanza IR, Rasbach KA, Okutsu M, Nair KS, Yan Z, Leinwand LA, Spiegelman BM. A PGC-1alpha isoform induced by resistance training regulates skeletal muscle hypertrophy. Cell 151: 1319-1331, 2012.

58. Sale DG, Jacobs I, MacDougall JD, Garner S. Comparison of two regimens of concurrent strength and endurance training. Med Sci Sports Exerc 22: 348-356, 1990.

59. Tesch PA, Ekberg A, Lindquist DM, Trieschmann JT. Muscle hypertrophy following 5-week resistance training using a non-gravity-dependent exercise system. Acta Physiol Scand 180: 89-98, 2004.
60. Thomson DM, Fick CA, Gordon SE. AMPK activation attenuates S6K1, $4 \mathrm{E}-\mathrm{BP} 1$, and eEF2 signaling responses to high-frequency electrically stimulated skeletal muscle contractions. J Appl Physiol 104: 625-632, 2008.

61. Wernbom M, Augustsson J, Thomee R. The influence of frequency, intensity, volume and mode of strength training on whole muscle crosssectional area in humans. Sports Med 37: 225-264, 2007.

62. Wilkinson SB, Phillips SM, Atherton PJ, Patel R, Yarasheski KE, Tarnopolsky MA, Rennie MJ. Differential effects of resistance and endurance exercise in the fed state on signalling molecule phosphorylation and protein synthesis in human muscle. J Physiol 586: 37013717, 2008.

63. Wilson JM, Marin PJ, Rhea MR, Wilson SM, Loenneke JP, Anderson JC. Concurrent training: a meta-analysis examining interference of aerobic and resistance exercises. J Strength Cond Res 26: 2293-2307, 2012.

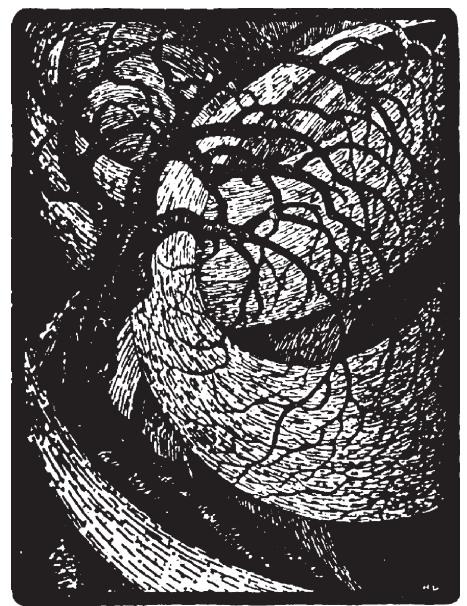

